\title{
14. Ueber die Spannung des gesättigten Wasserdampfes bei Temperaturen unter $0^{\circ}$; von M. Thiesen.
}

Die folgenden Rechnungen wurden zu dem Zwecke ausgefübrt, zu untersuchen, inwieweit wesentlich schärfere Bestimmungen der Spannung des Wasserdampfes, als sie bisher vorliegen, bei niedrigen Temperaturen von Interesse sein können. Zunächst wird die Temperatur bestimmt, bei welcher der Unterschied der Spannungen über Wasser und Eis ein Maximum wird, sodann werden auch die Spannungen unter $0^{0}$ für beide Fälle berechnet. Einige der sich dabei beiläufig ergebenden Beziehungen dürften nicht ohne Interesse sein.

Wir stellen nun zunächst die Bedingungsgleichung für die Lage des Maximums auf; dass ein solches überhaupt vorhanden sein muss, folgt daraus, dass der Unterschied sowohl in der Nähe von $0^{0}$ als auch bei sehr niederen Temperaturen verschwindet.

Sei $v_{1}$ und $v_{2}$ das Volumen des Dampfes und der Flüssigkeit bei der absoluten Temperatur $T, p$ der Druck des in Berührung mit der Flüssigkeit gesättigten Dampfes, $\varrho$ die Verdampfungswärme des Wassers, und seien die entsprechenden Grössen für Eis durch Indices unterschieden, so ist nach Clapeyron und Clausius

$$
\left(v_{1}-v_{2}\right) \frac{d p}{d T}=\frac{o}{T}
$$

(2) $\quad\left(v_{1}^{\prime}-v_{3}^{\prime}\right) \frac{d p^{\prime}}{d T}=\frac{\mathrm{g}^{\prime}}{T}$.

Für das Maximum des Spannungsunterschiedes verschwindet die Aenderung von $p-p^{\prime}$; es wird also

$$
\frac{v_{1}^{\prime}-v_{2}^{\prime}}{v_{1}-v_{2}}=\frac{\varrho^{\prime}}{\varrho} \text {. }
$$

In die bisher strengen Formeln werde jetzt eine weiterhin näher begründete Näherung durch die Annahme eingeführt, dass man dem Mariotte-Gay-Lussac'schen Gesetze entsprechend setzen könne

$$
p\left(v_{1}-v_{2}\right)=p^{\prime}\left(v_{1}^{\prime}-v_{2}^{\prime}\right)=R T .
$$


Die Gleichungen (1), (2), (3) gehen dann über in

$$
\begin{gathered}
\frac{d \log p}{d T}=\frac{\varrho}{R T^{2}} ; \quad \quad \quad \quad(6) \quad \frac{d \log p^{\prime}}{d T}=\frac{\varrho^{\prime}}{R T^{2}} ; \\
p^{\prime}=\frac{\varrho^{\prime}}{\varrho} .
\end{gathered}
$$

Bezeichnet $T_{0}$ die wenig über $0^{0}$ liegende Temperatur, für die $p^{\prime}=p$ wird, so ergiebt sich durch Subtraction von (5) und (6) nebst darauffolgender Integration

$$
\log \frac{p}{p^{\prime}}=-\int_{T_{0}}^{T} \frac{\varrho^{\prime}-\varrho}{R T^{2}} d T
$$

und durch Verbindung dieser Gleichung mit (7) schliesslich

$$
\log \frac{\varrho^{\prime}}{\varphi}=-\int_{T_{0}}^{T} \frac{\varrho^{\prime}-\varrho}{R T^{2}} d T .
$$

Aus dieser Gleichung lässt sich die Lage des Maximums genügend scharf berechnen; $\mathrm{zu}$ diesem Zwecke sind die einzelnen in die Gleichung eingehenden Grössen näher zu untersuchen.

Die Grösse $\varrho^{\prime}-\varrho$ ist die Schmelzwärme des Eises; sie möge mit $\sigma$ bezeichnet werden. Die Aenderung dieser Grösse mit der Temperatur bei - wie wir hier ohne wesentlichen Fehler annehmen können - constantem Drucke ist gegeben durch

$$
d \sigma=\left(C-C^{\prime}\right) d T
$$

falls $C$ und $C^{\prime}$ die specifischen Wärmen des Wassers und Eises bei dem constanten, verschwindend kleinen Drucke sind.

Die numerischen Werthe von $\sigma, C, C^{\prime}$ in der Nähe von $0^{0}$ sind - calorisch - gegeben durch

$$
\sigma_{0}=79,9 ; \quad C=1: \quad C^{\prime}=0,474 ;
$$

die Schmelzwärme wächst daher für jeden Grad um 0,526 oder 0,0066 ibres Werthes, fast ebenso schnell wie $T^{2}$; etwas genauer setzen wir

$$
\frac{\sigma}{T^{2}}=\frac{\sigma_{0}}{T^{2}}(1-0,0007 t),
$$


indem wir der Kürze wegen $t=T-T_{0}$ einführen. Die rechte Seite in Gleichung (8) erhält demnach den Werth

$$
-\frac{\sigma_{0}}{R T_{0}^{2}}(1-0,0004 t) t \text {. }
$$

Zur Berechnung der hier auftretenden Grösse $\sigma_{0} / R T_{0}^{2}$ schlagen wir zwei Wege ein.

Erstens verwandeln wir den obigen Werth von $\sigma_{0}$ durch Multiplication mit 41,34 (Atmosphäre als Druckeinheit) in mechanisches Maass und berechnen den Werth von $R$ aus dem entsprechenden Werth für Kohlensäure, wie er aus Regnault's Beobachtungen folgt ${ }^{1}$ ), durch Reduction mit den neuesten Atomgewichten. Wir erhalten so

$$
\frac{\sigma_{0}}{R T_{0}^{2}}=\frac{79,9 \cdot 41,34 \cdot 0,2200}{273^{2}}=0,00975 \text {. }
$$

Zweitens formen wir den soeben berechneten Ausdruck mittels Gleichung (5) in

$$
\frac{\sigma}{\varrho} \cdot \frac{d \log p}{d T}\left(\text { bei } 0^{0}\right)
$$

um. $\varrho$ berechnen wir für $0^{0}$ ans ${ }^{2}$ )

$$
\varrho=\mathfrak{r}(\mathfrak{T}-T) \frac{1}{3}
$$

mit $\left.\log \mathrm{r}=1,9214^{\mathrm{3}}\right), \quad \mathfrak{T}-T_{0}=365 \mathrm{zu} \varrho_{0}=596,3$ (calorisch). Zur Berechnung des zweiten Factors bediene ich mich der Gleichung - in der $t$ von $0^{0}$ an gerechnet ist -

(13) $T \log p=5,409(t-100)-0,508 \cdot 10^{-8}\left\{(365-t)^{4}-265^{4}\right\}$.

Die Gleichung, welche nur zwei aus Spannungsbeobachtungen zu bestimmende Constanten enthält, stellt die besten Spannungsbeobachtungen zwischen $0^{\circ}$ und $180^{\circ}$ gut dar; aus ihr folgt

$$
\left.\frac{d \log p}{d T}=0,07268 \text { (bei } 0^{0}\right) \text {. }
$$

Demnach wird zweitens

$$
\frac{\sigma_{0}}{R T_{0}^{2}}=\frac{79,9}{596,3} 0,07268=0,00974 .
$$

1) M. Thiesen, Wied. Ann. 24. p. 483. 1885.

2) M. Thiesen, Sitzungsber. der Physik. Gesellsch. zu Berlin 16. p. 80.1897 .

3) Ich bezeichne durchweg den natïrlichen Logarithmus mit log, den gemeinen mit Log. 
Die vollkommene Uebereinstimmung dieses Werthes mit dem obigen beweist die Gültigkeit des A vogadro'sehen Gesetzes bei $0^{\circ}$ und macht die Annahme der Gültigkeit des Mariotte'schen Gesetzes, unter der Gleichung (5) abgeleitet wurde, wahrscheinlich.

Der in Gleichung (8) noch auftretende Werth von $\varrho^{\prime}$ ergiebt sich als Summe von $\varrho$ und $\sigma$ bei $0^{\circ}$ gleich 676,2 . Seine Aenderung mit der Temperatur bei $0^{0}$ wird gleich

$$
-0,545+0,526=-0,019
$$

für jeden Grad und ist für den vorliegenden Zweck zu vernachlässigen. Sie ist wesentlich kleiner als die Aenderung der Dichte des Eises mit der Temperatur; eine noch grössere Constanz folgt aus der Uebereinstimmung der von Regnault beobachteten specifischen Wärmen des Eises und des Dampfes.

Fasst man nun alles zusammen, so nimmt die Bedingungsgleichung (8) eine solche Form an, dass $t$ einer sich nur langsam mit $t$ ändernden Grösse gleich wird. Man findet daher leicht für $t$ einen Näherungswerth und nach Berechnung der langsam variirenden Grösse für diesen Näherungswerth den genauen Werth von $t$. Die Ausfübrung der Rechnung ergiebt

$$
-t=\frac{\log \frac{676,2}{602,7}}{0,00975.1,005}=11,7^{\circ} \text {. }
$$

Die Extrapolation der in die Rechnung eingegangenen Grössen erstreckt sich demnach nur auf wenige Grade und der berechnete Werth kann als recht sicher angesehen werden. Fischer ${ }^{1}$ ) ist in seinen Beobachtungen dieser Temperatur bereits so nahe gekommen, dass er wohl bei niederer Temperatur keinen merklich grösseren Unterschied zwischen der Spannung des gesättigten Dampfes über Eis und Wasser beobachtet hätte.

Wir gehen jetzt zu dem Versuch über, die Spannungen selbst, und zwar zunächst die Spannung des Dampfes über dem Eise zu berechnen. Aus Gleichung (6) ergiebt sich durch Integration, wenn wir die oben gefundene annähernde Constanz von $\varrho^{\prime}$ als eine vollständige ansehen,

$$
\log \frac{p^{\prime}}{p_{0}}=\frac{\varrho^{\prime}}{R T_{0}} \frac{t}{T}
$$

1) W. Fischer, Wied. Ann. 28. p. 400. 1886. 
und wenn wir die oben gefundenen Werthe einsetzen und zum gemeinen Logarithmus übergehen

$$
\log \frac{p^{\prime}}{p_{0}}=9,78 \frac{t}{T} .
$$

Der Werth von Log $p_{0}$ ergiebt sich aus Gleichung (13) gleich - 2,2198 oder, falls wir jetzt zum Millimeter Quecksilber als Druckeinheit übergehen, zu $+0,6610$. Mit diesem Werthe sind mittels (16) die weiter unten folgenden Werthe von $p^{\prime}$ berechnet.

Für die Berechnung der Spannung des Dampfes über Wasser ist, um die Voraussetzungen dieses Aufsatzes beizubehalten, der Werth von $\varrho$ aus (12) in (5) einzuführen. Das Resultat der Integration der gewonnenen Gleichung lässt sich zwar in endlicher Form ausdrücken, doch ist eine Reihenentwickelung vorzuziehen. Für höhere Temperaturen wird das Resultat jedenfalls unrichtig, da dann nicht mehr das Mariotte'sche Gesetz gilt, für sehr tiefe ist es ohne Interesse, da es hier nicht mehr gelingt, das Wasser flüssig zu erhalten; wir geben daher der Entwickelung die Form, welche für T'emperaturen in der Nähe von $0^{\circ}$ am passendsten scheint, nämlich

(17) $\log \frac{p}{p_{0}}=\frac{\varrho_{0}}{R T_{0}} \frac{t}{T}\left(1-\frac{t}{6\left(\mathfrak{T}-T_{0}\right)}+\frac{3 \mathfrak{I}-5 T_{0}}{18 T_{0}\left(\mathfrak{T}-T_{0}\right)^{2}} t^{2}-\ldots\right)$,

oder nach Einsetzen der numerischen Werthe

(18) $\log \frac{p}{p_{0}}=\frac{t}{T}\left(8,628-0,00394 t+0,000002 t^{2}-\ldots\right)$.

Nach dieser Formel sind die unten angeführten Werthe von $p$ berechnet.

Wie vorhin angegeben, stellt die empirische Formel (13) sehr gut die Spannungsbeobachtungen bei höheren Temperaturen dar. Dieselbe für sehr tiefe Temperaturen anzuwenden, erscheint von vornherein misslich, da die Formel sich nicht gut mit der Annahme der Gültigkeit des Mariotte'schen Gesetzes vereinigen lässt. Immerhin ist es nöthig, zu untersuchen, inwieweit bei der Berechnung von $p$ nach den beiden Formeln (13) und (18) die Continuität praktisch gewahrt ist. Wir bringen daher (13) in dieselbe Form wie (18) und finden

$$
\log \frac{p}{p_{0}}=\frac{t}{T}\left(8,617-0,00406 t+0,000007 t^{2}-\ldots\right) \text {. }
$$


Der Unterschied zwischen den nach dieser und nach Formel (18) berechneten Werthen von $p$ erreicht in der Nähe von $t=-13,6^{\circ}$ ein Maximum ron etwa $0,0016 \mathrm{~mm}$ Quecksilber. In Ansehung der Genauigkeit der bisher vorliegenden Spannungsbeobachtungen kann man daher beide Formeln für Temperaturen unter $0^{\circ}$ als gleich ansehen.

Von den Annahmen, welche der Berechnung der folgenden Werthe zu Grunde liegen, ist wohl die Spannung bei $0^{\circ}$ am unsichersten. Fine selbst recht genaue Neubestimmung bei niedrigen Temperaturen dürfte kaum etwas anderes als eine Verbesserung dieses Werthes ergeben, der aber natürlich zweckmässiger direct zu bestimmen ist. Die Werthe von $p^{\prime}$ für sehr niedrige Temperaturen sind für Fragen der kosmischen Physik von Interesse.

Spannung des gesättigten Wasserdampfes in Millimeter Quecksilber

\begin{tabular}{lll}
\multicolumn{1}{c}{ bei } & über Eis & über Wasse \\
$t=$ & $p^{\prime}=4,581$ & $p=4,581$ \\
$t=-5$ & $p^{\prime}=3,010$ & $p=3,162$ \\
$t=-10$ & $p^{\prime}=1,946$ & $p=2,145$ \\
$t=-11,7$ & $p^{\prime}=1,672$ & $p=1,873$ \\
$t=-15$ & $p^{\prime}=1,237$ & $p=1,432$ \\
$t=-20$ & $p^{\prime}=0,772$ & $p=0,939$ \\
$t=-25$ & $p^{\prime}=0,473$ & $p=0,604$ \\
$t=-30$ & $p^{\prime}=0,284$ & \\
$t=-35$ & $p^{\prime}=0,167$ & \\
$t=-40$ & $p^{\prime}=0,096$ & \\
$t=-45$ & $p^{\prime}=0,054$ & \\
$t=-50$ & $p^{\prime}=0,029$ & \\
$t=-55$ & $p^{\prime}=0,016$ & \\
$t=-60$ & $p^{\prime}=0,008$ & \\
$t=-65$ & $p^{\prime}=0,004$ & \\
$t=-70$ & $p^{\prime}=0,002$ & \\
$t=-75$ & $p^{\prime}=0,0009$ & \\
$t=-80$ & $p^{\prime}=0,0004$ &
\end{tabular}

Friedrichshagen, den 12. Januar 1899.

(Eingegangen 14. Januar 1899.) 